\title{
Technetium-99m labelled liposomes to image experimental arthritis
}

Otto C Boerman, Wim J G Oyen, Gert Storm, M Luisa Corvo, Louis van Bloois, Jos W M van der Meer, Frans H M Corstens

\begin{abstract}
Objectives-Liposomes sterically stabilised with polyethylene glycol (PEG) labelled with technetium-99m were tested for their ability to image adjuvant arthritis in a rat model.

Methods-Adjuvant arthritis was induced in the ankle joint of the left hind foot by injection of Mycobacterium butyricum in Freund's incomplete adjuvant in the foot pad. Seven days later animals received the following radiopharmaceuticals labelled with ${ }^{99 m}$ Tc (a) non-PEG-liposomes, (b) PEG-liposomes or (c) non-specific human polyclonal IgG. For each of the radiopharmaceuticals the in vivo distribution of the radiolabel was monitored both scintigraphically as well as by counting the dissected tissues at two, eight, and 24 hours after injection.
\end{abstract}

Results-The pharmacokinetics of the radiopharmaceuticals differed considerably (half life in the blood: PEG-liposomes (18 hours) > ${ }^{99 m}$ Tc-IgG (3 hours) > non-PEG liposomes (1 hour)). The inflamed focus was visualised with each of the agents. The uptake of each of the radiopharmaceuticals in the inflamed ankle region correlated with their residence time in the blood (inflamed joint uptake: PEG liposomes (1.15\% injected dose (ID)/ g) $>{ }^{99 \mathrm{~m}}$ Tc-IgG $\quad(0.35 \% \quad$ ID/g $)>$ non-PEGliposomes $(0.05 \% \mathrm{ID} / \mathrm{g}))$. Quantitative analysis of the images showed that the inflamed ankle to background ratio was highest with the PEG-liposomes ( 7.5 at 24 hours after injection), while with the other two agents this ratio did not exceed 4 .

Conclusion-This study shows that ${ }^{99 \mathrm{~m}} \mathrm{Tc}$ labelled PEG-liposomes may be an excellent agent to visualise arthritis. Increased label uptake in the inflamed joint and increased target to background ratios can be obtained with PEG-liposomes because of their long circulating properties. In addition to their use as vehicles for scintigraphic imaging of arthritis PEGliposomes might also be used for the site specific delivery of antirheumatic drugs.

(Ann Rheum Dis 1997;56:369-373)

The clinical course of rheumatoid arthritis is highly variable, with most patients suffering from persistent but fluctuating disease activity and variable episodes of more active and more quiescent inflammation. The assessment of the inflammatory activity in the involved joints is important, not only for accurate early diagnosis, but also to monitor the state of the disease and to evaluate the effect of the therapeutic regimen. However, because considerable interobserver and intraobserver variability exists, there is a continued interest in the development of an objective, quantitative method to assess disease activity in the involved joints.

The use of radiopharmaceuticals in the detection of arthritis activity has the advantages of permitting direct imaging by means of whole body scintigraphy, while joints that are difficult to assess clinically or radiographically can be studied as well. A wide range of technetium-99m labelled radiopharmaceuticals has been proposed to visualise rheumatoid arthritis: nanocolloid, methylene diphosphonate (MDP), ${ }^{99 \mathrm{~m}} \mathrm{Tc}-\mathrm{IgG} \quad\left({ }^{99 \mathrm{~m}} \mathrm{Tc}-\right.$ labelled human immunoglobulin G). The conventional bone scan with ${ }^{99 \mathrm{~m}} \mathrm{Tc}-\mathrm{MDP}$ is a sensitive test to study bone diseases. It has been shown, however, that in patients presenting with rheumatoid arthritis bone scans frequently show increased uptake in the affected joints unrelated to disease activity. ${ }^{1-3}$ In the late 1980s liposomes have been tested to image rheumatoid arthritis. ${ }^{4}$ However, the recognition of these liposomes by cells of the mononuclear phagocyte system (MPS) caused relative high uptake in liver and spleen, concomitant with rapid blood clearance and low uptake in the target. In recent years, it has been shown that inclusion of polyethyleneglycol (PEG), conjugated to phosphatidylethanolamine, in the lipid bilayer, considerably decreased the recognition of the liposomes by MPS cells. ${ }^{5}$ It is postulated that the flexible and hydrophilic PEG-tails cause the formation of a water mantle around the liposomes. As a result the interaction of liposomes with macromolecules from the surrounding solution is reduced and thus the liposomes are protected from destruction or opsonisation and recognition by MPS cells. ${ }^{6}$ As a result PEG-liposomes have an increased residence time in the blood. ${ }^{7}$ In previous studies we have shown that radiolabelled PEG-liposomes accumulate in infectious and inflammatory foci ${ }^{8-10}$ because of the locally enhanced capillary permeability. The prolonged residence time in the blood enables the PEG-liposomes to extravasate to a relatively high degree at sites of enhanced vascular permeability. Therefore, radiolabelled PEG-liposomes may also have potential to visualise joints affected by rheumatoid 
arthritis. Moreover, PEG-liposomes can also be 'loaded' with drugs and thus could potentially be used for site directed delivery of antirheumatic drugs.

In this study we compared the imaging potential of ${ }^{99 \mathrm{~m}} \mathrm{Tc}$-PEG-liposomes with that of non-PEG-liposomes and the commercially available imaging agent ${ }^{99 \mathrm{~m}} \mathrm{Tc}-\mathrm{IgG}$, in rats with adjuvant arthritis.

\section{Methods}

ANIMAL MODEL

Adjuvant arthritis was induced in the left foot of randomly bred male Wistar rats (3 month old, body weight $300 \mathrm{~g}$ ) as described by Weichman. ${ }^{11}$ After ether anaesthesia, $0.1 \mathrm{ml}$ of a suspension of Mycobacterium butyricum (Difco Labs, Detroit, MI) in Freund's incomplete adjuvant (Sigma Chemical Co, St Louis, MO) $(10 \mathrm{mg} / \mathrm{ml})$ was injected in the foot pad of the left foot. Six days after induction, accumulation of mononuclear cells in the loose connective tissues of the foot can be appreciated at histological examination. Seven days after the inoculation, the respective radiopharmaceuticals were injected via the tail vein.

\section{PREPARATION OF LIPOSOMES}

Partially hydrogenated egg-phosphatidylcholine with an iodine value of 40 (PHEPC) prepared as described previously ${ }^{12}$ was obtained from Asahi Chemical Industry Co (Ibarakiken, Japan). Cholesterol and glutathione were obtained from Sigma (St Louis, MO). For the preparation of PEG-liposomes the polyethyleneglycol (PEG) 1900 derivative of DSPE (a kind gift of Sequus Inc, Menlo Park, CA), while for the preparation of the non-PEGliposomes underivitised DSPE (Avanti Polar lipids, Montreal,) was used as an ingredient.

A chloroform/methanol mixture $(10 / 1, \mathrm{v} / \mathrm{v})$ containing DSPE, PHEPC, and cholesterol was prepared at a molar ratio of $0.15: 1.85: 1$. A lipid film was formed by rotary evaporation followed by high vacuum to remove residual organic solvent. The lipid film was dispersed at room temperature in $50 \mathrm{mM}$ glutathione in HEPES buffer (10 mM HEPES, $135 \mathrm{mM}$ $\mathrm{NaCl}, \mathrm{pH} 7.5$ ) at a phospholipid concentration of $120 \mathrm{mM}$. The liposomes were sequentially extruded through polycarbonate filters of 200, 100,80 , and $50 \mathrm{~nm}$ pore size (Poretics, Livermore, CA). Unentrapped glutathione was removed by gel filtration on a $10 \mathrm{DG}$ column (Bio-Rad, Richmond, CA). The particle size distribution was determined by dynamic light scattering (Malvern 4700 system, Malvern, UK). The liposomes had a mean size of 120 $\mathrm{nm}$.

\section{LABELLING PROCEDURES}

Preformed glutathione containing non-PEGliposomes or PEG-liposomes were labelled with ${ }^{99 \mathrm{~m}} \mathrm{Tc}$ essentially as described previously. ${ }^{13}{ }^{99 \mathrm{~m}} \mathrm{Tc}$ was transported by $d, l$-hexamethylpropylene amine oxime (HMPAO) through the bilayer and trapped irreversibly in the internal aqueous phase caused by reduction by the encapsulated glutathione. Briefly, $2.0 \mathrm{ml}$ of liposomes (75 mmol phospholipid/ml) were incubated for 15 minutes at room temperature with $20 \mathrm{mCi}{ }^{99 m} \mathrm{Tc}-\mathrm{HMPAO}$. Removal of unencapsulated ${ }^{99 \mathrm{~m}} \mathrm{Tc}-\mathrm{HMPAO}$ was achieved by gel filtration on a $10 \mathrm{DG}$ column (Bio-Rad) eluted with $5 \%$ glucose.

A Technescan-HIG vial (Mallinckrodt Medical, Petten, the Netherlands) was reconstituted with $20 \mathrm{mCi}{ }^{99 m} \mathrm{Tc}$-pertechnetate according to the manufacturer's instructions. The resulting ${ }^{99 \mathrm{~m}} \mathrm{Tc}-\mathrm{IgG}$ was used without any further purification.

BIODISTRIBUTION STUDIES

Seven days after the inoculation of $M$ butyricum in the foot pad, 45 rats were divided randomly into three groups of 15 rats. Each group was injected with $100 \mu \mathrm{Ci}$ of either ${ }^{99 \mathrm{~m}} \mathrm{Tc}$-labelled non-PEG-liposomes, PEG-liposomes or ${ }^{99 \mathrm{~m}} \mathrm{Tc}-$ IgG via the tail vein.

At two, eight, and 24 hours after injection, five rats of each group were killed with $30 \mathrm{mg}$ intraperitoneally injected phenobarbital. Blood was obtained by cardiac puncture. After cervical dislocation, several tissues (inflamed left ankle, right ankle, muscle, liver, spleen, kidney, intestine, right femur, and bone marrow from the right femur) were dissected, weighed, and their activity was measured in a shielded well type gammacounter. To correct for physical decay and to permit calculation of the uptake of the radiopharmaceuticals in each organ as a fraction of the injected dose, aliquots of the injected dose were counted simultaneously. Inflamed ankle to contralateral ankle uptake ratios (IA/CA) were calculated.

IMAGING PROTOCOL

Adjuvant arthritis was induced in another nine rats as described above. Seven days later, groups of three rats received $300 \mu \mathrm{Ci}$ of ${ }^{99 \mathrm{~m}} \mathrm{Tc}$ labelled non-PEG-liposomes, PEG-liposomes or ${ }^{99 \mathrm{~m}} \mathrm{Tc}-\mathrm{IgG}$ via the tail vein. Rats were anaesthesised (halothane/nitrousoxide/oxygen) and were placed prone on a single head gammacamera equipped with a parallel hole low energy collimator (Orbiter, Siemens Inc, Hoffmann Estates, IL). The three groups of rats were imaged synchronously at five minutes, one, two, four, six, and 24 hours after injection. Symmetric $15 \%$ windows were used for the $140 \mathrm{keV}$ energy peak. Images (300 000 counts per image) were obtained and stored in a $256 \times 256$ matrix.

The scintigraphic results were analysed by drawing regions of interest over the heart region, the inflamed foot, over the normal contralateral foot-used as a background region -and over the whole animal. Inflamed foot to background ratios and percentage residual activity in the inflamed foot (inflamed foot to whole body ratio) were calculated.

STATISTICAL ANALYSIS

Values are given as mean (SD). Statistical analysis was performed using the one way analysis of variance test. 


\section{Results}

LABELLING AND QUALITY CONTROL OF THE RADIOPHARMACEUTICALS

The labelling efficiency of Technescan HIG 10 minutes after reconstitution with $20 \mathrm{mCi}$ ${ }^{99} \mathrm{TcO}_{4}$ was $97 \%$ as determined by instant thin layer chromatography (Gelman Labs, Ann Arbor, MI). The non-PEG and the PEG-liposomes were labelled with ${ }^{99 \mathrm{~m}} \mathrm{Tc}$ HMPAO with a labelling efficiency of $73 \%$ and $76 \%$, respectively. Analysis of a sample of each of the purified labelled liposome preparations on a BioRad 10DG column indicated that more than $96 \%$ of the radioactivity was associated with the liposomes.

BIODISTRIBUTION STUDIES

Table 1 shows the biodistributions of the ${ }^{99 m}$ Tc-labelled non-PEG-liposomes, PEGliposomes, and ${ }^{99 \mathrm{~m}} \mathrm{Tc}-\mathrm{IgG}$ in rats with adjuvant arthritis induced in the left foot. The non-PEG-liposomal preparation cleared very rapidly from the blood: two hours after injection blood concentrations had fallen to values as low as $0.17(0.03) \% \mathrm{ID} / \mathrm{g}$. These non-PEG-liposomes showed relatively high uptake in liver and spleen early after injection (2.65 (0.20) and $5.10 \quad(1.09) \% \quad \mathrm{ID} / \mathrm{g}$, respectively at two hours after injection). In contrast, blood clearance of the PEGliposomes was much slower (fig 1A). Blood concentrations of the ${ }^{99 \mathrm{~m}} \mathrm{Tc}-\mathrm{IgG}$ preparation were intermediate between those of the two liposomal preparations at all time points (fig 1A).

The three radiopharmaceuticals also showed considerable differences in uptake in the inflamed ankle (table 1). With the PEG-liposomes this uptake increased over time up to a value of $1.15(0.18) \% \mathrm{ID} / \mathrm{g}$ at 24 hours after injection, while the inflamed ankle uptake with the non-PEG-liposomes and the ${ }^{99 m}$ Tc-IgG remained almost at the same level throughout the study period $(0.1 \% \mathrm{ID} / \mathrm{g}$ and $0.5 \% \mathrm{ID} / \mathrm{g}$, respectively) as shown in figure $1 \mathrm{~B}$. At eight hours and 24 hours after injection the IA/CA ratio obtained with the PEG-liposomes was significantly higher than the ratio obtained with the other two radiopharmaceuticals $(\mathrm{p}<0.05)$. The IA/CA ratios obtained with the PEG-liposomes increased in time up to a value of 7.51 (0.91) (fig 2).
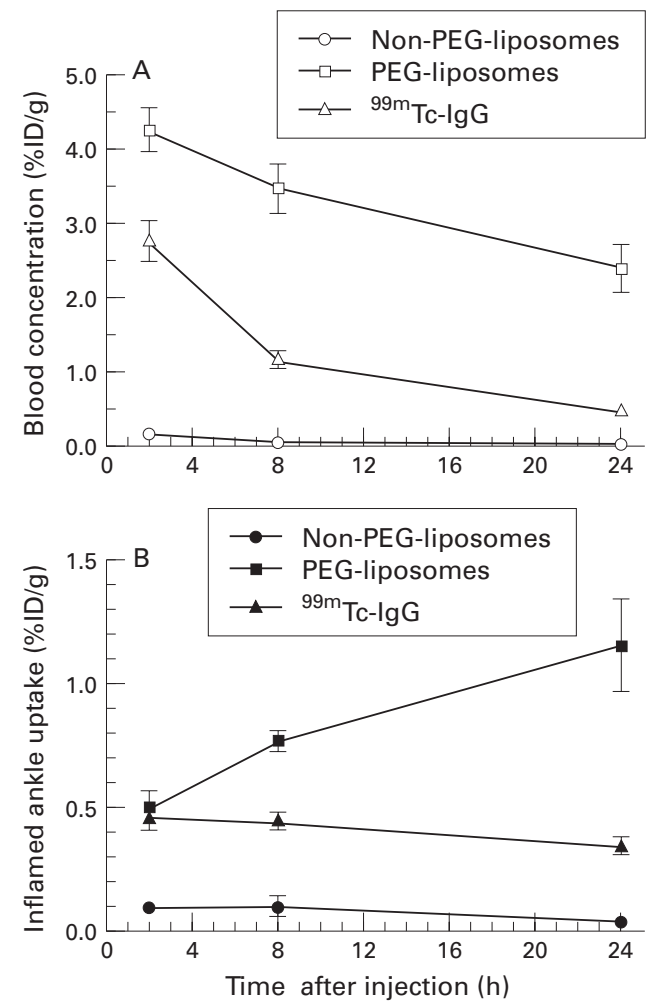

Figure 1 Blood concentration $(A)$ and uptake in the inflamed ankle (B) of the $99 m$ Tc-labelled non-PEG-liposomes, $P E G$-liposomes, and ${ }^{99 m} T c-I g G$ in rats with adjuvant arthritis in the left ankle. Five rats per group were used. Error bars represent $S D$.

Both liposomal formulations showed relatively high uptake of the radiolabel in the spleen. At 24 hours after injection splenic uptake was $4.0(0.8) \% \mathrm{ID} / \mathrm{g}$ and $11.1(1.5) \%$ ID/g for non-PEG and PEG-liposomes, respectively. With the ${ }^{99 \mathrm{~m}} \mathrm{Tc}-\mathrm{IgG}$ preparation uptake in the spleen was much lower (0.34 (0.04) \% ID/g, 24 hours after injection). With this radiopharmaceutical the kidney was the organ with the highest activity $(7.62(1.29) \%$ $\mathrm{ID} / \mathrm{g}, 24$ hours after injection).

IMAGING STUDIES

The inflamed foot was visualised with each of the radiopharmaceuticals included in this study. Figure 3 shows the image 24 hours after injection of the rats that received ${ }^{99 \mathrm{~m}} \mathrm{Tc}$-labelled non-PEG-liposomes, PEG-liposomes or

Table 1 Biodistribution of ${ }^{99 m}$ Tc-non-PEG-liposomes, ${ }^{99 m}$ Tc-PEG-liposomes, and ${ }^{99 m}$ Tc-IgG in rats with adjuvant arthritis two, eight, and 24 hours after injection (\%ID/g 5 rats/group)

\begin{tabular}{|c|c|c|c|c|c|c|c|c|c|}
\hline & \multicolumn{3}{|c|}{ Non-PEG-liposomes } & \multicolumn{3}{|c|}{ PEG-liposomes } & \multicolumn{3}{|l|}{${ }^{99 m} T c-I g G$} \\
\hline & 2 hours & 8 hours & 24 hours & 2 hours & 8 hours & 24 hours & 2 hours & 8 hours & 24 hours \\
\hline Blood & $0.17(0.03)$ & $0.07(0.01)$ & $0.03(0.01)$ & $4.27(0.28)$ & $3.49(0.33)$ & $2.42(0.33)$ & $2.79(0.27)$ & $1.19(0.10)$ & $0.50(0.09)$ \\
\hline Inflamed ankle & $0.10(0.01)$ & $0.11(0.04)$ & $0.05(0.01)$ & $0.49(0.08)$ & $0.77(0.04)$ & $1.15(0.18)$ & $0.46(0.05)$ & $0.44(0.05)$ & $0.35(0.03)$ \\
\hline Unaffected ankle & $0.03(0.00)$ & $0.03(0.01)$ & $0.02(0.00)$ & $0.14(0.02)$ & $0.14(0.02)$ & $0.16(0.02)$ & $0.16(0.01)$ & $0.11(0.02)$ & $0.08(0.01)$ \\
\hline Muscle & $0.01(0.00)$ & $0.01(0.00)$ & $0.00(0.00)$ & $0.05(0.01)$ & $0.06(0.02)$ & $0.04(0.01)$ & $0.05(0.01)$ & $0.05(0.02)$ & $0.02(0.01)$ \\
\hline Bone marrow & $0.29(0.08)$ & $0.25(0.09)$ & $0.24(0.07)$ & $0.72(0.17)$ & $0.83(0.09)$ & $1.03(0.16)$ & $0.76(0.08)$ & $0.56(0.12)$ & $0.32(0.05)$ \\
\hline Femur & $0.08(0.02)$ & $0.03(0.01)$ & $0.03(0.01)$ & $0.16(0.03)$ & $0.11(0.02)$ & $0.17(0.02)$ & $0.20(0.02)$ & $0.09(0.02)$ & $0.07(0.02)$ \\
\hline Lung & $0.16(0.05)$ & $0.09(0.02)$ & $0.07(0.02)$ & $0.95(0.12)$ & $1.08(0.23)$ & $0.70(0.07)$ & $0.94(0.22)$ & $0.57(0.12)$ & $0.27(0.05)$ \\
\hline Spleen & $5.01(1.09)$ & $5.04(1.21)$ & $4.01(0.80)$ & $6.15(0.47)$ & $7.84(0.95)$ & $11.1(1.46)$ & $0.87(0.11)$ & $0.55(0.09)$ & $0.34(0.04)$ \\
\hline Kidney & $3.68(0.45)$ & $3.56(0.71)$ & $2.97(0.43)$ & $1.41(0.24)$ & $1.73(0.12)$ & $2.60(0.36)$ & $7.84(0.79)$ & $7.80(0.90)$ & $7.62(1.29)$ \\
\hline Liver & $2.65(0.20)$ & $1.81(0.36)$ & $1.28(0.43)$ & $1.16(0.23)$ & $0.98(0.21)$ & $0.78(0.31)$ & $1.24(0.10)$ & $0.74(0.07)$ & $0.33(0.08)$ \\
\hline Intestine & $0.12(0.02)$ & $0.05(0.01)$ & $0.02(0.00)$ & $0.62(0.28)$ & $0.71(0.20)$ & $0.60(0.04)$ & $0.50(0.05)$ & $0.25(0.04)$ & $0.13(0.02)$ \\
\hline IA/UA ratio & $2.97(0.35)$ & $4.97(2.48)$ & $2.96(0.54)$ & $3.53(0.62)$ & $5.58(1.01)$ & $7.51(0.91)$ & $2.92(0.49)$ & $3.9(0.67)$ & $4.27(0.65)$ \\
\hline
\end{tabular}




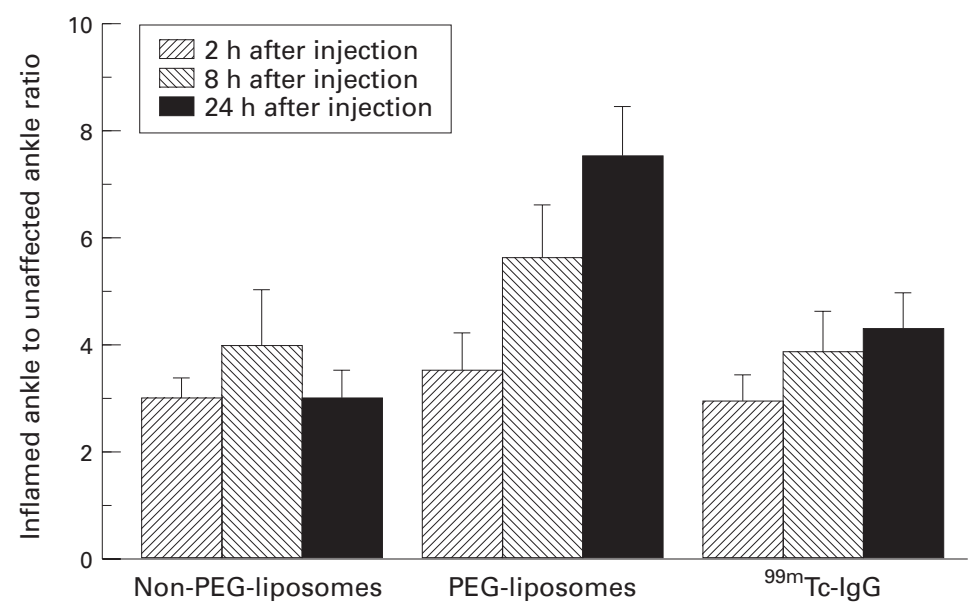

Figure 2 Inflamed ankle to unaffected ankle ratio obtained with non-PEG-liposomes, PEG-liposomes, and ${ }^{99 m} T c-I g G$ two hours, eight hours, and 24 hours after injection. The biodistribution data of five rats per group were used. Error bars represent SD.

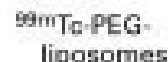

liposomes

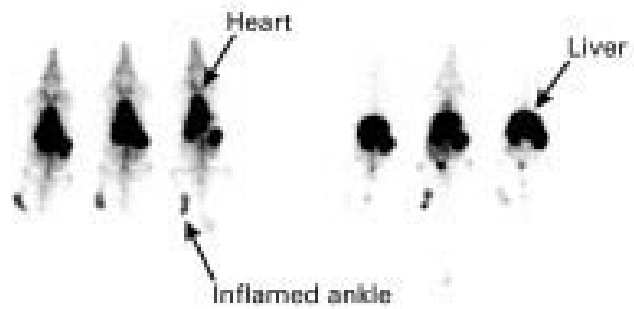

Figure 3 Scintigrams of rats with adjuvant arthritis imaged 24 hours after injection of ${ }^{99_{m}}$ Tc-labelled PEG-liposomes, non-PEG-liposomes, and ${ }^{99 m}$ Tc-IgG.

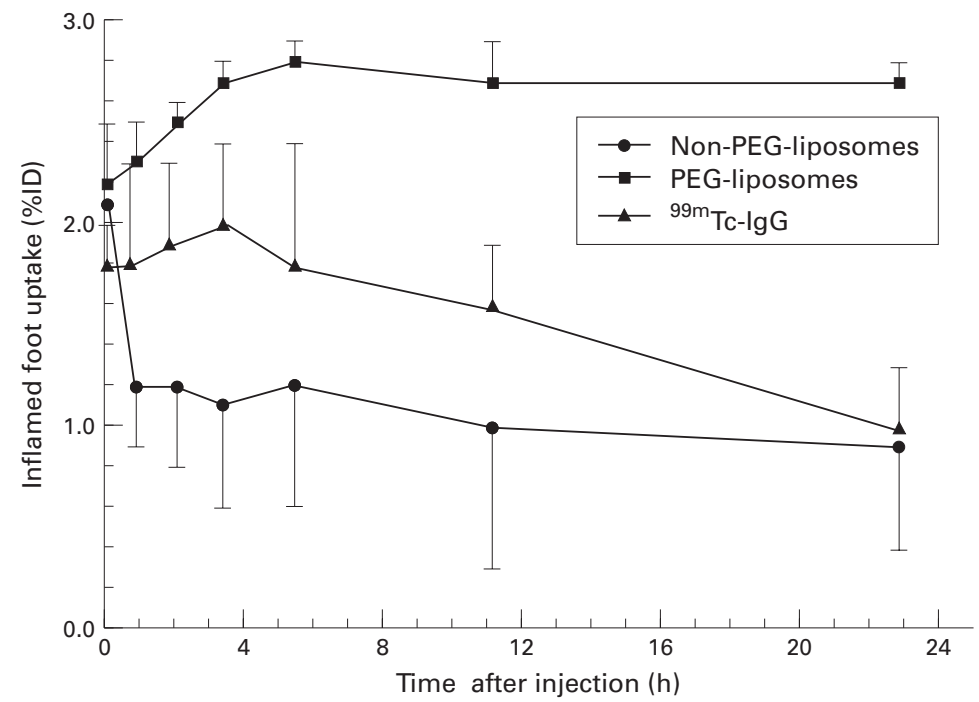

Figure 4 Quantitative analysis of the scintigraphic images of rats (three rats per group) injected with ${ }^{99_{m}}$ Tc-labelled non-PEG-liposomes, PEG-liposomes, and ${ }^{99_{m}} T c-I g G$. The activity in the inflamed foot was assessed. The whole body activity at five minutes after injection was set at $100 \%$ ID. Error bars represent SD.

${ }^{99 m}$ Tc-IgG. Quantitative analysis of the images clearly showed the main differences in circulatory half life of the three agents studied. The initial half life of the activity from the heart region of the PEG-liposomes, the non-PEG- liposomes, and the ${ }^{99 \mathrm{~m}} \mathrm{Tc}-\mathrm{IgG}$ was 18 hours, one hour, and three hours, respectively (data not shown). Furthermore, quantitative analysis of the images confirmed the superiority of the PEG-liposomes: from four hours onwards the uptake in the inflamed foot was significantly higher for the PEG-liposomes as compared with the other preparations ( $p<0.03$ ) (fig 4). Activity in the contralateral foot was low for each of the radiopharmaceuticals. Consequently inflamed foot to background ratios were highest for the PEG-liposomes as well (7.4 (1.1) $v \leq 3.6$ (1.5), 24 hours after injection).

\section{Discussion}

This study showed that in a rat model PEG-liposomes preferentially localise to arthritic joints, and thus PEG-liposomes potentially could be used (a) to image rheumatoid arthritis or (b) for the site specific delivery of antirheumatic drugs. The diagnosis of rheumatoid arthritis in patients with typically established disease is relatively easily made, but may be more difficult early in the course of the disease. An accurate clinical and diagnostic evaluation in patients is important because early treatment to suppress the inflammatory process may prevent progressive damage to articular structures. Furthermore, accurate assessment of the status of the disease allows a more objective way to evaluate the efficacy of the therapeutic regimen.

Liposomes, sterically stabilised with PEG, have been shown to preferentially localise to infectious foci. ${ }^{14}{ }^{15}$ In previous studies we have shown that sterically stabilised liposomes labelled with gamma-emitters can be used to image infectious and inflammatory foci in soft tissue..$^{8-10}$ This study in rats with adjuvant arthritis showed that ${ }^{99 \mathrm{~m}}$ Tc-PEG-liposomes are superior to both ${ }^{99 \mathrm{~m}} \mathrm{Tc}$-non-PEG-liposomes as well as ${ }^{99} \mathrm{~m}$ Tc-IgG to image experimental arthritis. The PEG-liposomes were superior in terms of both absolute uptake in the target tissue as well as target to background ratios. Several studies in patients with rheumatoid arthritis have shown that ${ }^{99} \mathrm{~m}$ Tc-IgG is a useful imaging agent for the clinical evaluation of rheumatoid arthritis, ${ }^{216} 17$ further supporting the clinical potential of ${ }^{99 \mathrm{~m}}$ Tc-labelled PEG-liposomes. In addition, the finding that PEG-liposomes displayed the highest absolute uptake in the inflammatory foci suggests that PEG-liposomes could also be exploited for site specific delivery of antirheumatic drugs. It has been shown in a rat model that liposomal methotrexate is more effective than the free drug in adjuvant arthritis. ${ }^{18}$ We have studied the therapeutic potential of using liposomes with encapsulated oxygen derived free radical scavengers in the model used in this study (Corvo et al, unpublished data).

The performance of the non-PEGliposomes in this study was comparable with the results obtained in previous studies: an optimised formulation of non-PEG-liposomes evaluated in a rat model of adjuvant arthritis revealed an initial half life of one to two hours, with an IA/CA ratio of $4 .{ }^{19}$ In clinical studies 
these non-PEG-liposomes labelled with ${ }^{99 \mathrm{~m}} \mathrm{Tc}$ localised to inflamed joints and not to non-affected joints. ${ }^{1420}$

Previous studies have shown that the interaction of PEG-liposomes with mononuclear cells in the circulation is minimal. Therefore, most probably the uptake of the PEG-liposomes in the inflammatory foci is mainly a result of extravasation caused by the locally increased vascular permeability. This would explain why uptake of the long circulating liposomes in the inflamed area is much higher than the uptake of the non-PEGliposomes: the persistently high blood concentrations of the PEG-liposomes facilitate the continued extravasation of the PEG-liposomes at the inflammatory focus. The mechanism of the subsequent retention of the liposomes in the inflammatory focus remains to be elucidated. The increased blood volume in the inflamed foot can only partly explain the increased uptake of PEG liposomes in the inflamed foot, because the IA/CA ratio increased with time. The IA/CA ratio obtained immediately after injection is a result of the increased vascularity in the inflamed foot and is relatively low.

Non-specific human polyclonal IgG has been shown to localise to foci of rheumatoid arthritis. ${ }^{21721}$ Initially it was proposed that the IgG was trapped specifically because of the interaction with $\mathrm{Fc}$ receptor positive inflammatory cells. $^{22}$ However, further studies have shown that the role of $\mathrm{Fc}$ receptor interaction in the accumulation of IgG in inflammatory foci is minimal. ${ }^{23}$ Therefore, scintigraphic detection of rheumatoid arthritis with either IgG or liposomes both represent non-specific targeting methods, exploiting the increased capillary permeability in inflammatory foci. Consequently, it is expected that imaging with a liposome based radiopharmaceutical cannot discriminate rheumatoid arthritis from other inflammatory processes in joints. The role of radiolabelled liposomes in the diagnostic investigation of patients with rheumatoid arthritis has to be determined in clinical studies.

In conclusion, this study shows that PEG-liposomes may be powerful vehicles for scintigraphic imaging of the joints affected by rheumatoid arthritis as well as for the targeted delivery of antirheumatic drugs. The skilled assistance of G Grutters (Central Animal Facility,
University Hospital Nijmegen) in the animal experiments and E University Hospital Nijmegen) in the animal experiments and $\mathrm{E}$ Hospital Nijmegen) in the preparation of the radiopharmaceuHospital Nijmegen) in the preparati
ticals is gratefully acknowledged.

1 O'Sullivan MM, Powell N, French AP, Williams KE, Morgan JR, Williams BD. Inflammatory joint disease: a gan JR, Williams BD. Inflammatory joint disease: a ography. Ann Rheum Dis 1988;47:485-91.
2 Berná L, Torres G, Diez C, Estorch M, Martinez-Duncker D, Carrio I. Technetium-99m human polyclonal immunoglobulin G Studies and conventional bone scans to noglobulin G Studies and conventional bone scans to detect active joint inflammation in chro
arthritis. Eur J Nucl Med 1992;19:173-6.

3 de Bois MH, Arndt JW, van der Velde EA, Pauwels EK. Joint scintigraphy for quantification of synovitis with ${ }^{99 m} \mathrm{Tc}$ labelled polyclonal human immunoglobulin $\mathrm{G}$ compared to late phase scintigraphy with ${ }^{99 \mathrm{~m}} \mathrm{Tc}$-labelled diphosphonate. Br J Rheumatol 1994;33:67-73.

4 Morgan JR, Williams LA, Howard CB. Technetium-labelled liposome imaging for deep-seated infection. $\mathrm{Br} \mathrm{J}$ Radiol 1985;58:35-9.

5 Boerman OC, Storm G, Oyen WJG, van Bloois L, van der Meer JWM, Claessens RAMJ, et al. Sterically stabilized liposomes labeled with ${ }^{111}$ In to image focal infection in
rats. J Nucl Med 1995; 36:1639-44.

6 Klibanov AL, Maruyama K, Torchilin VP, et al. Amphipathic polyethyleneglycols effectively prolong the circulation time of liposomes. FEBS Lett 1990;268:235-7.

7 Torchilin VP. How do polymers prolong circulation time of liposomes? J Lipid Res 1996; 99-116.

8 Allen TM, Hansen C, Martin F, Redemann C, Yan-Young A. Liposomes containing synthetic lipid derivatives of polyethylene glycol show prolonged circulation half-lives polyethylene glycol show prolonged circulation

9 Oyen WJG, Boerman OC, Storm G, van Bloois L, Koenders EB, Claessens RAMJ, et al. Detecting infection and inflammation with Technetium-99m stealth liposomes. J Nucl Med 1996;37:1392-7.

10 Oyen WJG, Boerman OC, Storm G, van Bloois L, Koenders EB, Crommelin DJA, et al. Labeled stealth liposomes in experimental infection: an alternative for leukocyte scintigraphy? Nucl Med Commun 1996;17:742-8.

11 Weichman BM. Rat adjuvant arthritis: a model of chronic inflammation. In:Chang JY, Lewis AJ, eds. Modern methods in pharmacology. Vol 5. New York: Alan R Liss, 1989: 36380 .

12 Lang J, Vigo-Pelfrey C, Martin F. Liposomes composed of partially hydrogenated egg phosphatidylcholines: fatty acid composition, thermal phase behaviour and oxidative stability. Chem Phys Lipids 1990;53:91-101.

13 Phillips WT, Rudolf AS, Goins B, Timmons JH, Klipper R, Blumhardt R. A simple method for producing a technetium-99m-labeled liposome which is stable in vivo. Nucl Med Biol 1992;19:539-47.

14 Bakker-Woudenberg IAJM, Lokerse AF, ten Kate MT, Storm G. Enhanced localization of liposomes with prolonged blood circulation time in infected lung tissue. prolonged blood circulation time in infected

15 Bakker-Woudenberg IAJM, Lokerse AF, ten Kate MT, Mouton JW, Woodle MC, Storm G. Liposomes with prolonged blood circulation and selective localization in Klebsiella pneumoniae-infected lung tissue. J Infect Dis 1993; 168:164-71.

16 Breedveld F, van Kroonenburgh MJPG, Camps JAJ, Feitsma HIJ, Markusse HM, Pauwels EKJ. Imaging of inflammatory arthritis with technetrium-99m-labeled IgG. J Nucl Med 1989;30:2017-21.

17 De Bois MH, Westedt ML, Arndt JW, Wiarda KS, van der Velde EA, et al. Technetium-99m labelled polyclonal human IgG scintigraphy before and 26 weeks after initiahuman IgG scintigraphy before and 26 weeks after initiation of parenteral gold treatment in patients with
toid arthritis. J Rheumatol 1995;22:1461-65.

18 Williams AS, Camilleri JP, Willems BD. Suppression of adjuvant-induced arthritis by liposomally conjugated methotrexate in the rat. Br J Rheumatol 1994;33:530-3.

19 Love WG, Amos N, Kellaway IW, Williams BD. Specific accumulation of cholesterol-rich liposomes in the inflammatory tissue of rats with adjuvant arthritis. Ann Rheum Dis 1990;49:611-4.

20 Williams BD, O'Sullivan MM, Saggu GS, Williams KE, Williams LA, Morgan JR. Synovial accumulation of technetium labelled liposomes in rheumatoid arthritis. Ann Rheum Dis 1987;46:314-8.

21 van der Lubbe PA, Arndt JW, Calame W, Ferreira TC, Pauwels EK, Breedveld FC. Measurement of synovial inflammation in rheumatoid arthritis with technetium $99 \mathrm{~m}$ labelled human polyclonal immunoglobulin G. Eur J Nucl Med 1991;18:118-23.

22 Rubin RH, Fischman AJ, Callahan RJ. In-111 labeled nonspecific immunoglobulin scanning in the detection of focal infection. N Engl J Med 1989;321:935-40.

23 Fischman AJ, Fucello AJ, Pellegrino-Gensey JL, Geltofsky J, Yarmush ML, Rubin RH, et al. Effect of carbohydrate modification on the localization of human polyclonal IgG at focal sites of bacterial infection. J Nucl Med 1992;33:1378-82. 